\title{
Increasing Nutritional Contents of Cassava Starch Wastes Using Pleurotus ostreatus (Jacq.) P.Kumm. and Lentinus squarrosulus Mont.
}

\author{
Kitti Wirunpan ${ }^{1 *}$, Sakesan Chinwang ${ }^{2}$, Nareerat Chaikong ${ }^{1}$ and Charida Pukahuta ${ }^{1}$ \\ ${ }^{1}$ Department of Biological Science, Faculty of Science, Ubon Ratchathani University, Ubon Ratchathani 34190, \\ Thailand. ${ }^{2}$ Department of Agriculture, Faculty of Agriculture, Ubon Ratchathani Rajabhat University. Ubon \\ Ratchathani 34000, Thailand.
}

\begin{abstract}
This research studied the increasing of nutritional content in cassava starch fermented with $P$. ostreatus (Jacq.) P.Kumm. and L. squarrosulus Mont. The cassava starch waste was supplemented with maize grain, paddy rice, rice bran, broken rice, and soybean meal at $0,10,20$, and $30 \%$. Solid state fermentation was carried out for 28 days. The results showed that highest reduced sugar at $86.09 \mathrm{~g} / \mathrm{L}$ was found in cassava starch waste $\mathbf{7 0} \%$ + rice broken $\mathbf{3 0} \%$ fermented by $L$. squarrosulus Mont. The highest protein content at $127.92 \mathrm{~g} / \mathrm{L}$ was found in cassava starch waste $90 \%$ + soybean meal $10 \%$ fermented with cassava starch waste $70 \%+$ rice broken $30 \%$ for 14 days. It is concluded from this experiment that $P$. ostreatus (Jacq.) P.Kumm. and L. squarrosulus Mont. can increase nutritional content in several feed mixtures.

Keywords: Cassava Starch Wastes, Pleurotus ostreatus (Jacq.) P.Kumm., nutritional content, Lentinus squarrosulus Mont.
\end{abstract}

\footnotetext{
*Correspondence: kitti.w@ubru.ac.th; +66-85-6325717

(Received: 10 November 2018; accepted: 22 December 2018)

Citation: Kitti Wirunpan, Sakesan Chinwang, Nareerat Chaikong and Charida Pukahuta, Increasing Nutritional Contents of Cassava Starch Wastes Using Pleurotus ostreatus (Jacq.) P.Kumm. and Lentinus squarrosulus Mont., J Pure App/ Microbiol., 2019; 13(1):117125 doi: 10.22207/JPAM.13.1.12

(c) The Author(s) 2019. Open Access. This article is distributed under the terms of the Creative Commons Attribution 4.0 International License which permits unrestricted use, sharing, distribution, and reproduction in any medium, provided you give appropriate credit to the original author(s) and the source, provide a link to the Creative Commons license, and indicate if changes were made.
} 


\section{INTRODUCTION}

It is estimated that the world cassava production in the year 2016 can be as high as 288 million tons. This is produced mainly in the continents of Africa, Asia and South America. The production in Thailand is estimated to be 3.2 million tons ${ }^{1}$ Thailand uses cassava for cassava flour (54\%), cassava chip (46\%) and ethanol production (2\%). By product of the cassava flour production is $10-15 \%$ cassava starch wastes, and this was used for animal feed but with some limitation due to high fiber and low protein contents ${ }^{2}$. Several methods were used to increase nutritional contents of this by-product. Some yeast and fungi species were used to increase the nutritional contents by fermentation ${ }^{3,4}$. Mushroom classified in the white rot fungi group possess ability to digest lignin and able to increase protein as found in Jonathan et al..$^{5}$. In these work, P. pulmonarius was used to cultivate with rice straw and sorghum stem. Protein content in rice straw was raised from $4.50 \%$ to $9.36 \%$ and $5.31 \%$ to $8.62 \%$ in the sorghum stem ${ }^{5}$, Bentil et al. also found that cocoa pulp protein content increased from $21 \%$ to $26 \%$ by using $P$. ostreatus for 6 weeks ${ }^{6}$. Darwish et al. also experiment by fermenting corn stem with $P$. ostreatus for 28 days and found that protein content was raised from $3.6 \%$ to $8.15 \%$. A work by Nasehi et al. using $P$. florida showed that protein content in rice straw was raised from $3.02 \%$ to $7.06 \%$ and that in wheat straw was raised from $3.71 \%$ to $7.38 \%^{8}$. This study was objected to determine the effect of a solid state fermentation involving $P$. ostreatus (Jacq.) P. Kumm. and $L$. squarrosulus Mont. on soluble protein and reducing sugars in cassava starch waste.

\section{MATERIALS AND METHODS}

\section{Experimental Design}

The experiment was laid out based on Completely Randomized Design (CRD) with 16 treatments and each treatment contain 3 replicates. This is as follow:

treatment 1 : controls (cassava starch waste $100 \%$ )

treatment 2 : cassava starch waste $90 \%$ + corn $10 \%$

treatment 3 : cassava starch waste $80 \%$ + corn $20 \%$

treatment 4 : cassava starch waste $70 \%$ $+\operatorname{corn} 30 \%$

treatment 5 : cassava starch waste $90 \%$ + paddy $10 \%$

treatment 6 : cassava starch waste $80 \%$ + paddy $20 \%$

treatment 7 : cassava starch waste $70 \%$ + paddy $30 \%$

treatment 8 : cassava starch waste $90 \%$

+ rice bran $10 \%$ treatment 9 : cassava starch waste $80 \%$

+ rice bran $20 \%$ treatment 10 : cassava starch waste $70 \%$ + rice bran $30 \%$ treatment 11 : cassava starch waste $90 \%$ + rice broken $10 \%$ treatment 12 : cassava starch waste $80 \%$ + rice broken $20 \%$ treatment 13 : cassava starch waste $70 \%$ + rice broken $30 \%$ treatment 14 : cassava starch waste $90 \%$ + soybean meal $10 \%$ treatment 15 : cassava starch waste $80 \%$ + soybean meal $20 \%$ treatment 16 : cassava starch waste $70 \%$ + soybean meal $30 \%$

The experiment is carried out in $15 \mathrm{x}$ $100 \mathrm{~mm}$ petri dishes. Material from treatment $1-16$ at $30 \mathrm{~g}$ was placed inside the dishes and autoclave at 121 degree Celsius for 60 minutes. The mycelia from P. ostreatus (Jacq.) P. Kumm. and $L$. squarrosulus Mont. was sub-cultured and stored at 27 and 35 degree Celsius, respectively for 14 and 28 days.

\section{Chemical analysis}

Reduced sugar: The amount of reduced sugar present in the samples was determined using DNS methodology. The absorbance at $530 \mathrm{~nm}$ was measured using a spectrophotometer ${ }^{9}$.

Soluble protein: The soluble protein concentrations were evaluated using a colorimetric method based on the standard curve of bovine serum albumin at $750 \mathrm{~nm}^{10}$.

Carbon content: The carbon content was determined following the rapid wet oxidation method $^{11}$.

\section{RESULTS AND DISCUSSION \\ Reduced sugar}

Reduced sugar content in various substrates was found to be significantly different 
$(P<0.05)$ after fermentation with $P$. ostreatus (Jacq.) P.Kumm. for 14 days (Table 1). Treatment 13 (cassava starch waste $70 \%+$ rice broken 30 $\%)$ showed the highest reduced sugar reading at $16.99 \mathrm{~g} / \mathrm{L}$. The lowest reduced sugar content was found in treatment 15 (cassava starch waste $80 \%$ + soybean meal $20 \%$ ).

Reduced sugar contents was also found to be significantly different $(P<0.05)$ after fermentation with $P$. ostreatus (Jacq.) P.Kumm. for 28 days (Table 2). At this period, treatment 10 (cassava starch waste $70 \%$ + rice bran $30 \%$ ) showed the highest reduced sugar content $(20.28$ $\mathrm{g} / \mathrm{L}$ ). Treatment 16 (cassava starch waste $70 \%+$ soybean meal $30 \%$ ) was found to contain the lowest reduced sugar reading $(0.69 \mathrm{~g} / \mathrm{L})$.

Reduced sugar content in all treatments was also found to be significantly different $(P<0.05)$ after fermentation with $L$. squarrosulus Mont. for 14 days (Table 1). Treatment 12 (cassava starch waste $70 \%$ + rice broken $30 \%$ ) showed the highest reduced sugar reading at $22.87 \mathrm{~g} / \mathrm{L}$. The lowest reduced sugar content was found in treatment 15 (cassava starch waste $80 \%+$ soybean meal $20 \%$ ).

Reduced sugar contents was also found to be significantly different $(P<0.05)$ after

Table 1. Effect of P. ostreatus (Jacq.) P.Kumm. and L. squarrosulus Mont. on reduced sugar content at 14 days (g/L)

\begin{tabular}{|c|c|c|c|c|c|}
\hline \multirow[b]{2}{*}{ Type of substrate } & \multirow{2}{*}{$\begin{array}{l}\text { Control } \\
14 \text { day }\end{array}$} & \multicolumn{3}{|c|}{ P. ostreatus (Jacq.) P.Kumm. } & \multirow{2}{*}{$\begin{array}{l}\text { L. squarrosulus Mont } \\
\text { Increasing }\end{array}$} \\
\hline & & 14day & Increasing & 14 day & \\
\hline & $\mathrm{g} / \mathrm{L}$ & $\mathrm{g} / \mathrm{L}$ & $\mathrm{g} / \mathrm{L}$ & $\mathrm{g} / \mathrm{L}$ & $\mathrm{g} / \mathrm{L}$ \\
\hline cassava starch waste $100 \%$ & $0.75^{\operatorname{defg}}$ & $14^{b}$ & $13.26^{\mathrm{a}}$ & $7.17^{\mathrm{bc}}$ & $6.42^{\mathrm{cd}}$ \\
\hline $\begin{array}{l}\text { cassava starch waste } \\
90 \%+\text { corn } 10 \%\end{array}$ & $1.25^{\text {cdef }}$ & $15.61^{\mathrm{ab}}$ & $14.37^{\mathrm{a}}$ & $11.29^{\mathrm{abc}}$ & $10.04^{\mathrm{abcd}}$ \\
\hline $\begin{array}{l}\text { cassava starch waste } \\
80 \%+\text { corn } 20 \%\end{array}$ & $1.32^{\text {cde }}$ & $14.63^{b}$ & $13.31^{\mathrm{a}}$ & $15.65^{\mathrm{abc}}$ & $14.34^{\mathrm{abcd}}$ \\
\hline $\begin{array}{l}\text { cassava starch waste } \\
70 \%+\text { corn } 30 \%\end{array}$ & $1.53^{\mathrm{bcd}}$ & $15.82^{\mathrm{ab}}$ & $14.29^{\mathrm{a}}$ & $13.76^{\mathrm{abc}}$ & $12.23^{\mathrm{abcd}}$ \\
\hline $\begin{array}{l}\text { cassava starch waste } \\
90 \%+\text { paddy } 10 \%\end{array}$ & $0.69^{\text {defg }}$ & $13.65^{b}$ & $12.97^{\mathrm{a}}$ & $8.65^{\mathrm{bc}}$ & $7.97^{\mathrm{bcd}}$ \\
\hline $\begin{array}{l}\text { cassava starch waste } \\
80 \%+\text { paddy } 20 \%\end{array}$ & $0.39^{\mathrm{fg}}$ & $14.91^{b}$ & $14.52^{\mathrm{a}}$ & $16.89^{\mathrm{abc}}$ & $16.50^{\mathrm{abcd}}$ \\
\hline $\begin{array}{l}\text { cassava starch waste } \\
70 \%+\text { paddy } 30 \%\end{array}$ & $0.21^{\mathrm{g}}$ & $16.93^{\mathrm{ab}}$ & $16.72^{\mathrm{a}}$ & $20.89^{\mathrm{ab}}$ & $20.67^{a b c}$ \\
\hline $\begin{array}{l}\text { cassava starch waste } \\
90 \%+\text { rice bran } 10 \%\end{array}$ & $3.19^{\mathrm{a}}$ & $16.48^{\mathrm{ab}}$ & $13.28^{\mathrm{a}}$ & $14.66^{\mathrm{abc}}$ & $11.48^{\mathrm{abcd}}$ \\
\hline $\begin{array}{l}\text { cassava starch waste } \\
80 \%+\text { rice bran } 20 \%\end{array}$ & $3.93^{\mathrm{a}}$ & $19.24^{\mathrm{a}}$ & $15.31^{\mathrm{a}}$ & $17.75^{\mathrm{abc}}$ & $13.83^{\mathrm{abcd}}$ \\
\hline $\begin{array}{l}\text { cassava starch waste } \\
70 \%+\text { rice bran } 30 \%\end{array}$ & $3.79^{a}$ & $8.36^{c}$ & $4.57^{b}$ & $17.26^{\mathrm{abc}}$ & $13.47^{\mathrm{abcd}}$ \\
\hline $\begin{array}{l}\text { cassava starch waste } \\
90 \%+\text { rice brokens } 10 \%\end{array}$ & $0.56^{\mathrm{efg}}$ & $14.75^{\mathrm{b}}$ & $14.19^{\mathrm{a}}$ & $22.82^{\mathrm{ab}}$ & $22.27^{a b c}$ \\
\hline $\begin{array}{l}\text { cassava starch waste } \\
80 \%+\text { rice brokens } 20 \%\end{array}$ & $0.36^{\mathrm{fg}}$ & $15.53^{\mathrm{ab}}$ & $15.17^{\mathrm{a}}$ & $26.20^{\mathrm{a}}$ & $25.84^{\mathrm{a}}$ \\
\hline $\begin{array}{l}\text { cassava starch waste } \\
70 \%+\text { rice brokens } 30 \%\end{array}$ & $0.35^{\mathrm{fg}}$ & $17.34^{\mathrm{ab}}$ & $16.99^{\mathrm{a}}$ & $23.21^{\mathrm{ab}}$ & $22.87^{a b}$ \\
\hline $\begin{array}{l}\text { cassava starch waste } \\
90 \%+\text { soybean meal } 10 \%\end{array}$ & $1.59^{b c d}$ & $6.01^{c d}$ & $4.43^{b}$ & $11.82^{\mathrm{abc}}$ & $10.23^{\mathrm{abcd}}$ \\
\hline $\begin{array}{l}\text { cassava starch waste } \\
80 \%+\text { soybean meal } 20 \%\end{array}$ & $2.27^{b}$ & $2.35^{d}$ & $0.075^{b}$ & $2.885^{c}$ & $0.61^{d}$ \\
\hline $\begin{array}{l}\text { cassava starch waste } \\
70 \%+\text { soybean meal } 30 \%\end{array}$ & $2.12^{\mathrm{bc}}$ & $2.66^{d}$ & $0.53^{b}$ & $3.785^{c}$ & $1.67^{d}$ \\
\hline
\end{tabular}

$a, b, c, d, e, f, g=$ Means in the same column with different superscripts are significantly different $(P=0.05)$ 
Wirunpan et al. J Pure Appl Microbiol, 13(1), 117-125 | March 2019 | DOI 10.22207/JPAM.13.1.12

Table 2. Effect of $P$. ostreatus (Jacq.) P.Kumm. and L. squarrosulus Mont. on reduced sugar content at 28 day (g/L)

\begin{tabular}{|c|c|c|c|c|c|}
\hline \multirow[b]{2}{*}{ Type of substrate } & \multirow{2}{*}{$\begin{array}{l}\text { Control } \\
28 \text { day }\end{array}$} & \multicolumn{3}{|c|}{ P. ostreatus (Jacq.) P.Kumm. } & \multirow{2}{*}{$\begin{array}{l}\text { L. squarrosulus Mont. } \\
\text { Increasing }\end{array}$} \\
\hline & & 28day & Increasing & 28 day & \\
\hline & $\mathrm{g} / \mathrm{L}$ & $\mathrm{g} / \mathrm{L}$ & $\mathrm{g} / \mathrm{L}$ & $\mathrm{g} / \mathrm{L}$ & $\mathrm{g} / \mathrm{L}$ \\
\hline cassava starch waste $100 \%$ & $0.66^{b}$ & $8.61^{\mathrm{e}}$ & $7.95^{f}$ & $8.82^{\mathrm{hi}}$ & $8.16^{\mathrm{ghi}}$ \\
\hline $\begin{array}{l}\text { cassava starch waste } \\
90 \%+\operatorname{corn} 10 \%\end{array}$ & $1.46^{\mathrm{b}}$ & $12.03^{\mathrm{de}}$ & $10.58^{\mathrm{ef}}$ & $31.6^{\mathrm{e}}$ & $30.14^{d}$ \\
\hline $\begin{array}{l}\text { cassava starch waste } \\
80 \%+\text { corn } 20 \%\end{array}$ & $1.69^{b}$ & $16.89^{b c}$ & $15.2^{\mathrm{bcd}}$ & $52.09^{c}$ & $50.41^{c}$ \\
\hline $\begin{array}{l}\text { cassava starch waste } \\
70 \%+\text { corn } 30 \%\end{array}$ & $1.76^{\mathrm{b}}$ & $13.26^{\mathrm{cd}}$ & $11.5^{\text {def }}$ & $62.76^{\mathrm{b}}$ & $61^{b}$ \\
\hline $\begin{array}{l}\text { cassava starch waste } \\
90 \%+\text { paddy } 10 \%\end{array}$ & $0.39^{b}$ & $13.9^{c d}$ & $13.52^{\text {bcde }}$ & $21.1^{\mathrm{fg}}$ & $20.71^{\text {def }}$ \\
\hline $\begin{array}{l}\text { cassava starch waste } \\
80 \%+\text { paddy } 20 \%\end{array}$ & $0.44^{\mathrm{b}}$ & $11.86^{\mathrm{de}}$ & $11.42^{\text {def }}$ & $42.86^{d}$ & $42.42^{c}$ \\
\hline $\begin{array}{l}\text { cassava starch waste } \\
70 \%+\text { paddy } 30 \%\end{array}$ & $0.26^{b}$ & $13.82^{\mathrm{cd}}$ & $13.57^{\text {bcde }}$ & $47.2^{\text {cd }}$ & $46.95^{c}$ \\
\hline $\begin{array}{l}\text { cassava starch waste } \\
90 \%+\text { rice bran } 10 \%\end{array}$ & $1.36^{b}$ & $18.67^{b}$ & $17.32^{\mathrm{ab}}$ & $16.52^{\mathrm{gh}}$ & $15.17^{\mathrm{efgh}}$ \\
\hline $\begin{array}{l}\text { cassava starch waste } \\
80 \%+\text { rice bran } 20 \%\end{array}$ & $1.79^{b}$ & $18.82^{b}$ & $17.03^{a b c}$ & $18.18^{g}$ & $16.39^{\mathrm{efg}}$ \\
\hline $\begin{array}{l}\text { cassava starch waste } \\
70 \% \text { + rice bran } 30 \%\end{array}$ & $5.17^{\mathrm{a}}$ & $25.44^{\mathrm{a}}$ & $20.28^{a}$ & $28.19^{e f}$ & $23.02^{\mathrm{de}}$ \\
\hline $\begin{array}{l}\text { cassava starch waste } \\
90 \%+\text { rice brokens } 10 \%\end{array}$ & $0.67^{b}$ & $15.97^{\mathrm{bcd}}$ & $15.3^{\mathrm{bcd}}$ & $63.22^{\mathrm{b}}$ & $62.56^{\mathrm{b}}$ \\
\hline $\begin{array}{l}\text { cassava starch waste } \\
80 \%+\text { rice brokens } 20 \%\end{array}$ & $0.33^{b}$ & $16.08^{\mathrm{bcd}}$ & $15.76^{\mathrm{bc}}$ & $80.41^{a}$ & $80.09^{a}$ \\
\hline $\begin{array}{l}\text { cassava starch waste } \\
70 \%+\text { rice brokens } 30 \%\end{array}$ & $0.32^{\mathrm{b}}$ & $13.51^{\mathrm{cd}}$ & $13.19^{\text {cde }}$ & $86.41^{a}$ & $86.09^{a}$ \\
\hline $\begin{array}{l}\text { cassava starch waste } \\
90 \%+\text { soybean meal } 10 \%\end{array}$ & $1.14^{\mathrm{b}}$ & $2.15^{f}$ & $1.01^{\mathrm{g}}$ & $14.47^{\mathrm{gh}}$ & $13.33^{\mathrm{fghi}}$ \\
\hline $\begin{array}{l}\text { cassava starch waste } \\
80 \%+\text { soybean meal } 20 \%\end{array}$ & $1.3^{b}$ & $2.72^{f}$ & $1.42^{\mathrm{g}}$ & $5.13^{i}$ & $3.84^{i}$ \\
\hline $\begin{array}{l}\text { cassava starch waste } \\
70 \%+\text { soybean meal } 30 \%\end{array}$ & $1.84^{b}$ & $2.53^{f}$ & $0.69^{g}$ & $8.57^{\mathrm{hi}}$ & $6.73^{\mathrm{hi}}$ \\
\hline
\end{tabular}

$a, b, c, d, e, f, g, h, i=$ Means in the same column with different superscripts are significantly different $(P=0.05)$

fermentation with $P$. ostreatus (Jacq.) P.Kumm. for 28 days (Table 2). At this period, treatment 13 (cassava starch waste $70 \%+$ rice broken 30 $\%)$ showed the highest reduced sugar content $(86.09 \mathrm{~g} / \mathrm{L})$. Treatment 15 (cassava starch waste $80 \%+$ soybean meal $20 \%$ ) was found to contain the lowest reduced sugar reading $(3.84 \mathrm{~g} / \mathrm{L})$.

Reduced sugar content in every treatment increased as the fermentation time increased. Hossain et al. also found reduced sugar content increasing after using $P$. sajor-caju co-cultivated with bean shell, rice straw, wheat straw, and sugarcane bagasse ${ }^{12}$. Adamafio et al. also found that cellulose was double after fermenting corn cob with $P$. ostreatus. Solid state fermentation with $P$. ostreatus decrease accumulated fiber by enzymes activities ${ }^{13}$. It is indicated that mushroom contains several enzymes that involves lignocellulose metabolism ${ }^{14}$. A similar finding was also found by Darwish et al. which used $P$. ostreatus and Saccharomyces cerevisiae with corn stem $^{7}$. Bentil et al. also found a similar result when experimented with cocoa pulp. 


\section{Carbon content}

Carbon content in every treatment showed significantly different $(P<0.05)$ after fermentation for 14 days (Table 3 ). It was found that cassava starch waste $70 \%+$ corn $30 \%$ showed the lowest reduction in carbon content (5.63\%). It was also found that a substrate mixture of cassava starch waste $70 \%$ + rice broken $20 \%$ showed the lowest reduction in carbon content $(0.11 \%)$. At day 28 after fermentation, the results were also significantly different $(P<0.05)$ (Table 4$)$. The mixture of cassava starch waste $70 \%+$ soybean meal $30 \%$ found the highest reduction in carbon content $(6.21 \%)$ and the mixture of cassava starch waste $90 \%$ + rice broken $10 \%$ resulted in the lowest reduction in carbon content $(0.14 \%)$.

\section{Protein contents}

Protein content percentage in all treatments showed significantly different $(P<0.05)$ after fermentation for 14 days (Table 5). It was found that protein content in cassava starch waste $70 \%+$ soybean meal $30 \%$ showed the highest

Table 3. Effect of P. ostreatus (Jacq.) P.Kumm. and L. squarrosulus Mont. on carbon percentage content at 14 day (g/L)

\begin{tabular}{|c|c|c|c|c|c|}
\hline \multirow{3}{*}{ Type of substrate } & \multirow{2}{*}{$\begin{array}{l}\text { Control } \\
14 \text { day }\end{array}$} & \multicolumn{4}{|c|}{ P. ostreatus (Jacq.) P.Kumm. L.squarrosulus Mont. } \\
\hline & & 14day & reduction & 14 day & reduction \\
\hline & $\%$ & $\%$ & $\%$ & $\%$ & $\%$ \\
\hline $\begin{array}{l}\text { cassava starch waste } \\
90 \%+\text { corn } 10 \%\end{array}$ & $47.74^{\mathrm{ab}}$ & $42.68^{a b c}$ & $5.06^{\mathrm{ab}}$ & $47.11^{\mathrm{ab}}$ & $0.63^{\mathrm{a}}$ \\
\hline $\begin{array}{l}\text { cassava starch waste } \\
80 \%+\text { corn } 20 \%\end{array}$ & $47.62^{a b c}$ & $43.27^{a b c}$ & $4.36^{a b c}$ & $47.42^{\mathrm{a}}$ & $0.20^{a}$ \\
\hline $\begin{array}{l}\text { cassava starch waste } \\
70 \%+\text { corn } 30 \%\end{array}$ & $48.33^{\mathrm{ab}}$ & $42.70^{a b c}$ & $5.63^{a}$ & $46.34^{\mathrm{ab}}$ & $2.00^{\mathrm{a}}$ \\
\hline $\begin{array}{l}\text { cassava starch waste } \\
90 \%+\text { paddy } 10 \%\end{array}$ & $47.06^{a b c}$ & $46.48^{a}$ & $0.58^{b c}$ & $45.99^{a b}$ & $1.06^{\mathrm{a}}$ \\
\hline $\begin{array}{l}\text { cassava starch waste } \\
80 \%+\text { paddy } 20 \%\end{array}$ & $45.4^{\mathrm{bcd}}$ & $44.84^{a b c}$ & $0.57^{\mathrm{bc}}$ & $43.45^{\mathrm{abcd}}$ & $1.95^{\mathrm{a}}$ \\
\hline $\begin{array}{l}\text { cassava starch waste } \\
70 \%+\text { paddy } 30 \%\end{array}$ & $46.42^{\mathrm{abc}}$ & $43.92^{a b c}$ & $2.50^{\mathrm{abc}}$ & $43.91^{\mathrm{abcd}}$ & $2.51^{a}$ \\
\hline $\begin{array}{l}\text { cassava starch waste } \\
90 \%+\text { rice bran } 10 \%\end{array}$ & $48.25^{\mathrm{ab}}$ & $45.34^{a b c}$ & $2.91^{\mathrm{abc}}$ & $44.44^{\mathrm{abc}}$ & $3.81^{\mathrm{a}}$ \\
\hline $\begin{array}{l}\text { cassava starch waste } \\
80 \%+\text { rice bran } 20 \%\end{array}$ & $46.62^{\mathrm{abc}}$ & $46.11^{\mathrm{ab}}$ & $0.51^{b c}$ & $42.83^{\mathrm{abcd}}$ & $3.78^{a}$ \\
\hline $\begin{array}{l}\text { cassava starch waste } \\
70 \%+\text { rice bran } 30 \%\end{array}$ & $48.50^{\mathrm{a}}$ & $46.11^{\mathrm{ab}}$ & $2.39^{\mathrm{abc}}$ & $44.62^{\mathrm{ab}}$ & $3.88^{a}$ \\
\hline $\begin{array}{l}\text { cassava starch waste } \\
90 \% \text { + rice broken } 10 \%\end{array}$ & $47.63^{\mathrm{abc}}$ & $46.32^{\mathrm{a}}$ & $1.31^{\mathrm{abc}}$ & $46.96^{\mathrm{ab}}$ & $0.67^{a}$ \\
\hline $\begin{array}{l}\text { cassava starch waste } \\
80 \%+\text { rice broken } 20 \%\end{array}$ & $46.25^{\mathrm{abcd}}$ & $46.15^{a b}$ & $0.11^{c}$ & $45.73^{a b}$ & $0.53^{\mathrm{a}}$ \\
\hline $\begin{array}{l}\text { cassava starch waste } \\
70 \%+\text { rice broken } 30 \%\end{array}$ & $48.4^{a}$ & $45.58^{a b}$ & $2.84^{\mathrm{abc}}$ & $44.64^{\mathrm{ab}}$ & $3.77^{\mathrm{a}}$ \\
\hline $\begin{array}{l}\text { cassava starch waste } \\
90 \%+\text { soybean meal } 10 \%\end{array}$ & $44.65^{c d}$ & $44.18^{a b c}$ & $0.48^{\mathrm{bc}}$ & $42^{b c d}$ & $2.65^{a}$ \\
\hline $\begin{array}{l}\text { cassava starch waste } \\
80 \%+\text { soybean meal } 20 \%\end{array}$ & $43.33^{\text {de }}$ & $41.19^{b c}$ & $2.15^{\mathrm{abc}}$ & $39.36^{c d}$ & $3.98^{\mathrm{a}}$ \\
\hline $\begin{array}{l}\text { cassava starch waste } \\
70 \%+\text { soybean meal } 30 \%\end{array}$ & $41.03^{e}$ & $40.29^{c}$ & $0.75^{\mathrm{bc}}$ & $38.82^{d}$ & $2.21^{\mathrm{a}}$ \\
\hline
\end{tabular}

$a, b, c, d=$ Means in the same column with different superscripts are significantly different $(P=0.05)$ 
Wirunpan et al. J Pure Appl Microbiol, 13(1), 117-125 | March 2019 | DOI 10.22207/JPAM.13.1.12

Table 4. Effect of $P$. ostreatus (Jacq.) P.Kumm. and L. squarrosulus Mont. on carbon percentage content at 28 day (g/L)

\begin{tabular}{|c|c|c|c|c|c|}
\hline \multirow{3}{*}{ Type of Substrate } & \multirow{2}{*}{$\frac{\text { Control }}{24 \text { day }}$} & \multicolumn{4}{|c|}{ P. ostreatus (Jacq.) P.Kumm. L. squarrosulus Mont. } \\
\hline & & 28day & reduction & 28 day & reduction \\
\hline & $\%$ & $\%$ & $\%$ & $\%$ & $\%$ \\
\hline $\begin{array}{l}\text { cassava starch waste } \\
90 \%+\text { corn } 10 \%\end{array}$ & $49.02^{\mathrm{a}}$ & $45.38^{b c}$ & $3.64^{\mathrm{ab}}$ & $47.82^{\mathrm{a}}$ & $1.20^{\mathrm{bc}}$ \\
\hline $\begin{array}{l}\text { cassava starch waste } \\
80 \%+\text { corn } 20 \%\end{array}$ & $48.83^{\mathrm{ab}}$ & $47.36^{\mathrm{ab}}$ & $1.47^{\mathrm{bc}}$ & $46.68^{\mathrm{ab}}$ & $2.15^{\mathrm{abc}}$ \\
\hline $\begin{array}{l}\text { cassava starch waste } \\
70 \%+\text { corn } 30 \%\end{array}$ & $49.29^{a}$ & $47.68^{a}$ & $1.61^{b c}$ & $45.98^{\mathrm{abc}}$ & $3.32^{\mathrm{abc}}$ \\
\hline $\begin{array}{l}\text { cassava starch waste } \\
90 \%+\text { paddy } 10 \%\end{array}$ & $46.04^{\text {cde }}$ & $45.67^{a b c}$ & $0.37^{c}$ & $45.2^{\mathrm{abcd}}$ & $0.84^{c}$ \\
\hline $\begin{array}{l}\text { cassava starch waste } \\
80 \%+\text { paddy } 20 \%\end{array}$ & $45.39^{\mathrm{de}}$ & $44.49^{c}$ & $0.90^{c}$ & $43.84^{c d}$ & $1.55^{b c}$ \\
\hline $\begin{array}{l}\text { cassava starch waste } \\
70 \%+\text { paddy } 30 \%\end{array}$ & $44.81^{\text {ef }}$ & $44.04^{c}$ & $0.77^{c}$ & $40.47^{f}$ & $4.35^{\mathrm{ab}}$ \\
\hline $\begin{array}{l}\text { cassava starch waste } \\
90 \%+\text { rice bran } 10 \%\end{array}$ & $47.99^{\mathrm{abcd}}$ & $46.76^{\mathrm{ab}}$ & $1.23^{b c}$ & $45.10^{\mathrm{bcd}}$ & $2.89^{a b c}$ \\
\hline $\begin{array}{l}\text { cassava starch waste } \\
80 \%+\text { rice bran } 20 \%\end{array}$ & $48.02^{\mathrm{abcd}}$ & $45.87^{a b c}$ & $2.15^{\mathrm{bc}}$ & $43.52^{c d}$ & $4.50^{\mathrm{ab}}$ \\
\hline $\begin{array}{l}\text { cassava starch waste } \\
70 \%+\text { rice bran } 30 \%\end{array}$ & $48.17^{a b c}$ & $45.95^{a b c}$ & $2.22^{\mathrm{bc}}$ & $43.19^{\text {de }}$ & $4.98^{\mathrm{a}}$ \\
\hline $\begin{array}{l}\text { cassava starch waste } \\
90 \%+\text { rice broken } 10 \%\end{array}$ & $47.62^{\mathrm{abcd}}$ & $47.47^{\mathrm{ab}}$ & $0.15^{c}$ & $46.68^{\mathrm{ab}}$ & $0.94^{c}$ \\
\hline $\begin{array}{l}\text { cassava starch waste } \\
80 \%+\text { rice broken } 20 \%\end{array}$ & $46.3^{\text {bcde }}$ & $45.42^{b c}$ & $0.88^{c}$ & $46.07^{a b c}$ & $0.23^{c}$ \\
\hline $\begin{array}{l}\text { cassava starch waste } \\
70 \%+\text { rice broken } 30 \%\end{array}$ & $48.16^{\mathrm{abc}}$ & $47.23^{\mathrm{ab}}$ & $0.93^{c}$ & $43.65^{\mathrm{cd}}$ & $4.51^{\mathrm{ab}}$ \\
\hline $\begin{array}{l}\text { cassava starch waste } \\
90 \%+\text { soybean meal } 10 \%\end{array}$ & $42.26^{\mathrm{bcd}}$ & $40.88^{d}$ & $1.38^{\mathrm{bc}}$ & $40.74^{\mathrm{ef}}$ & $1.51^{b c}$ \\
\hline $\begin{array}{l}\text { cassava starch waste } \\
80 \%+\text { soybean meal } 20 \%\end{array}$ & $41.99^{\mathrm{g}}$ & $36.52^{\mathrm{e}}$ & $5.47^{a}$ & $39.21^{f}$ & $2.78^{a b c}$ \\
\hline $\begin{array}{l}\text { cassava starch waste } \\
70 \%+\text { soybean meal } 30 \%\end{array}$ & $40.80^{\mathrm{g}}$ & $34.60^{\mathrm{e}}$ & $6.21^{\mathrm{a}}$ & $39.37^{f}$ & $1.43^{b c}$ \\
\hline
\end{tabular}

$a, b, c, d, e, f, g=$ Means in the same column with different superscripts are significantly different $(P=0.05)$

protein content $(69.92 \mathrm{mg} / \mathrm{g})$. The mixture of cassava starch waste $70 \%+$ rice bran $30 \%$ showed the lowest protein content $(3.93 \mathrm{mg} / \mathrm{g})$. It was also showed significantly different $(P<0.05)$ after fermentation for 28 days (Table 6). The highest protein content $(51.43)$ were found in cassava starch waste $70 \%+$ soybean meal $30 \%$ and the lowest protein content were found in cassava starch waste $80 \%+$ paddy $20 \%$ ( $8.31 \mathrm{mg} / \mathrm{g})$.

The experiment with $L$. squarrosulus Mont. for 14 days also showed significantly different $(\mathrm{P}<0.05)$ after fermentation for 14 days (Table 5). The highest protein content (127.92 $\mathrm{mg} / \mathrm{g}$ ) was found in cassava starch waste $90 \%$ + soybean meal $10 \%$. The protein content in cassava starch waste $90 \%$ + paddy $10 \%$ showed the lowest percentage $(3.40 \mathrm{mg} / \mathrm{g})$. At day 28 after fermentation the result also showed significantly different $(P<0.05)$ (Table 6). Cassava starch waste $80 \%+$ soybean meal $20 \%$ gave the highest protein content $(100.52 \mathrm{mg} / \mathrm{g})$. The lowest protein content (16.65 mg/g) was found in cassava starch $100 \%$.

The result in this experiment clearly showed that both species of white rot fungi can increase protein content and other nutrients in cassava starch waste. An experiment by Akinifemi 
et $\mathrm{al}^{15}$ using $P$. ostreatus and $P$. pulmonarius cocultivate with sorghum for 21 days showed that protein content increased from 2.54 to 4.54 and 4.59 respectively, the cocoa pulp nutrient increased when this was fermented with $P$. ostreatus for 6 weeks ${ }^{6,16}$. Bentil et al. study the influence of white rot fungi ( $P$. ostreatus) on chemical compositions in agro-industrial residues and found that protein content sawdust and bagasse increased from $0.91 \%$ to $2.49 \%$ and $0.39 \%$ and $2.99 \%$, respectively ${ }^{16}$. Shrivastava et al. using P. ostreatus on sorghum straw and found protein content increased from $3.37 \%$ to $5.08 \%{ }^{17}$. It was also found that carbon to nitrogen ratio decreased from 77.6 to 40.45 when compared to control without fermentation ${ }^{7}$. Experiment on corn stem using $P$. ostreatus by fermentation for 28 days and found that total protein was increased from $3.6 \%$ to $8.15 \%$. Protein was increased from mycelium and released enzymes ${ }^{12}$.

Table 5. Effect of P. ostreatus (Jacq.) P.Kumm. and L. squarrosulus Mont. on protein content at 14 day (g/L)

\begin{tabular}{|c|c|c|c|c|c|}
\hline Type of substrate & $\begin{array}{c}\text { Control } \\
14 \text { day } \\
\text { g/L }\end{array}$ & $\begin{array}{c}\text { P. ostre } \\
\text { 14day } \\
\mathrm{g} / \mathrm{L}\end{array}$ & $\begin{array}{c}\text { (Jacq.) P.Kur } \\
\text { Increasing } \\
\text { g/L }\end{array}$ & $\begin{array}{l}\text { L. squarr } \\
14 \text { day } \\
\text { g/L }\end{array}$ & $\begin{array}{c}\text { sulus Mont. } \\
\text { Increasing } \\
\mathrm{g} / \mathrm{L}\end{array}$ \\
\hline cassava starch waste $100 \%$ & $3.75^{d}$ & $9.51^{f}$ & $5.91^{\mathrm{cd}}$ & $11.14^{\mathrm{d}}$ & $7.39^{d}$ \\
\hline $\begin{array}{l}\text { cassava starch waste } \\
90 \%+\text { corn } 10 \%\end{array}$ & $4.63^{d}$ & $19.28^{\text {de }}$ & $10.88^{\text {cd }}$ & $20.03^{d}$ & $15.41^{d}$ \\
\hline $\begin{array}{l}\text { cassava starch waste } \\
80 \%+\text { corn } 20 \%\end{array}$ & $6.88^{d}$ & $21.21^{\text {cde }}$ & $11.23^{\mathrm{cd}}$ & $22.16^{d}$ & $15.28^{d}$ \\
\hline $\begin{array}{l}\text { cassava starch waste } \\
70 \%+\text { corn } 30 \%\end{array}$ & $5.98^{\mathrm{d}}$ & $21.83^{\text {cde }}$ & $11.51^{\text {cd }}$ & $27.23^{d}$ & $21.25^{d}$ \\
\hline $\begin{array}{l}\text { cassava starch waste } \\
90 \%+\text { paddy } 10 \%\end{array}$ & $7.48^{\mathrm{d}}$ & $14.29^{\mathrm{ef}}$ & $7.83^{\mathrm{cd}}$ & $10.88^{d}$ & $3.40^{d}$ \\
\hline $\begin{array}{l}\text { cassava starch waste } \\
80 \%+\text { paddy } 20 \%\end{array}$ & $5.40^{d}$ & $15.36^{\mathrm{ef}}$ & $7.69^{\mathrm{cd}}$ & $17.91^{d}$ & $12.51^{d}$ \\
\hline $\begin{array}{l}\text { cassava starch waste } \\
70 \%+\text { paddy } 30 \%\end{array}$ & $4.98^{d}$ & $13.54^{\mathrm{ef}}$ & $5.68^{\text {cd }}$ & $21.82^{d}$ & $16.84^{d}$ \\
\hline $\begin{array}{l}\text { cassava starch waste } \\
90 \%+\text { rice bran } 10 \%\end{array}$ & $13.13^{\mathrm{cd}}$ & $26.28^{c d}$ & $9.48^{\mathrm{cd}}$ & $32.76^{d}$ & $19.64^{d}$ \\
\hline $\begin{array}{l}\text { cassava starch waste } \\
80 \%+\text { rice bran } 20 \%\end{array}$ & $21.69^{c}$ & $28.59^{c}$ & $7.25^{\mathrm{cd}}$ & $98.96^{c}$ & $77.27^{d}$ \\
\hline $\begin{array}{l}\text { cassava starch waste } \\
70 \%+\text { rice bran } 30 \%\end{array}$ & $19.85^{c}$ & $28.54^{c}$ & $3.94^{d}$ & $114.17^{c}$ & $94.31^{\mathrm{bc}}$ \\
\hline $\begin{array}{l}\text { cassava starch waste } \\
90 \%+\text { rice broken } 10 \%\end{array}$ & $6.21^{d}$ & $14.93^{\mathrm{ef}}$ & $9.47^{c d}$ & $21.70^{d}$ & $15.49^{d}$ \\
\hline $\begin{array}{l}\text { cassava starch waste } \\
80 \%+\text { rice broken } 20 \%\end{array}$ & $3.96^{d}$ & $19.22^{\text {de }}$ & $13.22^{\mathrm{c}}$ & $23.35^{d}$ & $19.39^{d}$ \\
\hline $\begin{array}{l}\text { cassava starch waste } \\
70 \%+\text { rice broken } 30 \%\end{array}$ & $3.92^{d}$ & $19.30^{\text {de }}$ & $12.80^{c d}$ & $22.93^{d}$ & $19.02^{d}$ \\
\hline $\begin{array}{l}\text { cassava starch waste } \\
90 \%+\text { soybean meal } 10 \%\end{array}$ & $42.50^{\mathrm{ab}}$ & $83.13^{b}$ & $51.05^{b}$ & $170.42^{\mathrm{a}}$ & $127.92^{\mathrm{a}}$ \\
\hline $\begin{array}{l}\text { cassava starch waste } \\
80 \%+\text { soybean meal } 20 \%\end{array}$ & $37.08^{b}$ & $108.03^{a}$ & $61.36^{\mathrm{a}}$ & $146.04^{b}$ & $108.96^{\mathrm{ab}}$ \\
\hline $\begin{array}{l}\text { cassava starch waste } \\
70 \%+\text { soybean meal } 30 \%\end{array}$ & $53.13^{a}$ & $116.88^{a}$ & $62.92^{\mathrm{a}}$ & $153.33^{\mathrm{ab}}$ & $100.21^{b}$ \\
\hline
\end{tabular}

$a, b, c, d, e, f=$ Means in the same column with different superscripts are significantly different $(P=0.05)$ 
Table 6. Effect of P. ostreatus (Jacq.) P.Kumm. and L. squarrosulus Mont. on protein content at 28 day (g/L)

\begin{tabular}{|c|c|c|c|c|c|}
\hline Type of Substrate & $\begin{array}{c}\text { Control } \\
28 \text { day } \\
\text { g/L }\end{array}$ & $\begin{array}{l}\text { P. ostreat } \\
\text { 28day } \\
\text { g/L }\end{array}$ & $\begin{array}{c}\text { (Jacq.) P.Kum } \\
\text { Increasing } \\
\text { g/L }\end{array}$ & $\begin{array}{l}\text { L. squar } \\
28 \text { day } \\
\text { g/L }\end{array}$ & $\begin{array}{c}\text { sulus Mont. } \\
\text { Increasing } \\
\mathrm{g} / \mathrm{L}\end{array}$ \\
\hline cassava starch waste $100 \%$ & $3.04^{h}$ & $13.08^{d}$ & $8.55^{d}$ & $19.69^{\mathrm{g}}$ & $16.65^{\mathrm{g}}$ \\
\hline $\begin{array}{l}\text { cassava starch waste } \\
90 \%+\text { corn } 10 \%\end{array}$ & $6.54^{\mathrm{ef}}$ & $20.11^{d}$ & $12.00^{\text {cd }}$ & $45.59^{\mathrm{ef}}$ & $39.04^{\mathrm{efg}}$ \\
\hline $\begin{array}{l}\text { cassava starch waste } \\
80 \%+\text { corn } 20 \%\end{array}$ & $7.21^{\mathrm{e}}$ & $19.49^{d}$ & $11.24^{\text {cd }}$ & $68.66^{\mathrm{de}}$ & $61.45^{\text {cde }}$ \\
\hline $\begin{array}{l}\text { cassava starch waste } \\
70 \%+\text { corn } 30 \%\end{array}$ & $7.31^{\mathrm{e}}$ & $23.11^{d}$ & $14.13^{\mathrm{bcd}}$ & $103.21^{\mathrm{ab}}$ & $95.90^{\mathrm{ab}}$ \\
\hline $\begin{array}{l}\text { cassava starch waste } \\
90 \%+\text { paddy } 10 \%\end{array}$ & $4.44^{\mathrm{fgh}}$ & $17.75^{d}$ & $9.90^{d}$ & $23.18^{\mathrm{fg}}$ & $18.74^{\mathrm{g}}$ \\
\hline $\begin{array}{l}\text { cassava starch waste } \\
80 \%+\text { paddy } 20 \%\end{array}$ & $5.46^{\mathrm{efg}}$ & $16.56^{d}$ & $8.89^{d}$ & $27.49^{\mathrm{fg}}$ & $22.03^{\mathrm{g}}$ \\
\hline $\begin{array}{l}\text { cassava starch waste } \\
70 \%+\text { paddy } 30 \%\end{array}$ & $4.69^{\mathrm{fgh}}$ & $18.03^{d}$ & $11.57^{c d}$ & $36.79^{\mathrm{fg}}$ & $32.10^{\mathrm{fg}}$ \\
\hline $\begin{array}{l}\text { cassava starch waste } \\
90 \%+\text { rice bran } 10 \%\end{array}$ & $12.88^{\mathrm{d}}$ & $26.02^{\mathrm{d}}$ & $13.52^{\mathrm{cd}}$ & $62.87^{\text {de }}$ & $49.99^{\text {def }}$ \\
\hline $\begin{array}{l}\text { cassava starch waste } \\
80 \%+\text { rice bran } 20 \%\end{array}$ & $15.50^{c}$ & $51.34^{d}$ & $32.15^{\mathrm{abc}}$ & $66.05^{\mathrm{de}}$ & $50.55^{\text {def }}$ \\
\hline $\begin{array}{l}\text { cassava starch waste } \\
70 \%+\text { rice bran } 30 \%\end{array}$ & $18.52^{b}$ & $60.46^{b c}$ & $35.69^{\mathrm{ab}}$ & $95.53^{\mathrm{abc}}$ & $77.01^{\mathrm{abc}}$ \\
\hline $\begin{array}{l}\text { cassava starch waste } \\
90 \%+\text { rice broken } 10 \%\end{array}$ & $5.35^{\mathrm{efgh}}$ & $20.74^{d}$ & $16.55^{\mathrm{bcd}}$ & $44.18^{\mathrm{efg}}$ & $38.82^{\mathrm{efd}}$ \\
\hline $\begin{array}{l}\text { cassava starch waste } \\
80 \%+\text { rice broken } 20 \%\end{array}$ & $3.98^{\mathrm{gh}}$ & $23.24^{d}$ & $17.57^{\mathrm{bcd}}$ & $77.49^{c d}$ & $73.51^{\mathrm{bcd}}$ \\
\hline $\begin{array}{l}\text { cassava starch waste } \\
70 \%+\text { rice broken } 30 \%\end{array}$ & $3.06^{h}$ & $23.50^{d}$ & $16.38^{\mathrm{bcd}}$ & $79.01^{\mathrm{bcd}}$ & $75.94^{\mathrm{abc}}$ \\
\hline $\begin{array}{l}\text { cassava starch waste } \\
90 \%+\text { soybean meal } 10 \%\end{array}$ & $18.00^{\mathrm{b}}$ & $54.38^{c}$ & $29.17^{\mathrm{bcd}}$ & $82.09^{\mathrm{bcd}}$ & $64.09^{c d}$ \\
\hline $\begin{array}{l}\text { cassava starch waste } \\
80 \%+\text { soybean meal } 20 \%\end{array}$ & $19.23^{b}$ & $81.41^{\mathrm{ab}}$ & $11.20^{\text {cd }}$ & $119.75^{\mathrm{a}}$ & $100.52^{\mathrm{a}}$ \\
\hline $\begin{array}{l}\text { cassava starch waste } \\
70 \%+\text { soybean meal } 30 \%\end{array}$ & $23.88^{a}$ & $98.10^{a}$ & $51.43^{\mathrm{a}}$ & $116.31^{\mathrm{a}}$ & $92.43^{\mathrm{ab}}$ \\
\hline
\end{tabular}

$a, b, c, d, e, f, g, h=$ Means in the same column with different superscripts are significantly different $(P=0.05)$

\section{CONCLUSION}

We conclude that Pleurotus ostreatus (Jacq.) P.Kumm. and Lentinus squarrosulus Mont. can increase protein content, and reduced sugar in the substrates with significantly different $(P<0.05)$. Carbon percentage was decreased after fermented with both species. The substrates were able to be used in livestock feed.

\section{ACKNOWLEDGEMENTS}

The authors thank Assistant Professor Dr. Sureerat Butprom for her kind technical advice and valuable comments on the manuscript.

\section{CONFLICT OF INTEREST}

The authors declares that there is no conflict of interest.

\section{REFERENCES}

1. FAO. Food outlook biannual report on global food markets. Food and Agriculture Organization of the United Nations., 2016; pp 74.

2. Khempaka, S., Molee, W., Guillaume, M. Dried cassava pulp as an alternative feedstuff for broilers: Effect on growth performance, carcass traits, digestive organs, and nutrient digestibility. J. Appl. Poult. Res., 2009; 18(3): 487-93.

3. Khempaka, S., Thongkratok, R., Okrathok, S., Molee, $W$. An evaluation of cassava pulp feedstuff fermented 
with A. oryzae, on growth performance, nutrient digestibility and carcass quality of broilers. J. Poult. Sci., 2014; 51(1): 71-9.

4. Oboh, G. Nutrient enrichment of cassava peels using a mixed culture of Saccharomyces cerevisae and Lactobacillus spp solid media fermentation techniques. Electron. J. Biotechno., 2006; 9(1); 46-9.

5. Jonathan, S.G., Okorie, A.N., Garuba, E.O., Babayemi, O.J. Bioconversion of sorghum stalk and rice straw into value added ruminant feed using Pleurotus pulmonarius. Nat. Sci., 2012; 10(4): 10-6.

6. Bentil, J.A.: Enhancement of the nutritive value of cocoa (Theobroma cacao) bean shells for use as feed for animals through a two-stage solid state fermentation with Pleurotus ostreatus and Aspergillus niger. Master of Science, Kwame Nkrumah University of Science and Technology, 2012; pp 82-3.

7. Darwish, G.A., Bakr, A.A., Abdallah, M.M.F. Nutritional value upgrading of maize stalk by using Pleurotus ostreatus and Saccharomyces cerevisiae in solid state fermentation. Ann. Agri. Sci., 2012; 57(1): 47-51.

8. Nasehi, M., Torbatinejad, N.M., Zerehdaran, S., Safaie, A.R. Effect of solid-state fermentation by oyster mushroom (Pleurotus florida) on nutritive value of some agro by-products. J. Appl. Anim. Res., 2017; 45(1): 221-6.

9. Miller, G. L. Use of dinitrosalicylic acid reagent for determination of reducing sugar. Anal. Chem., 1959; 31(3): 426-8.

10 Lowry, O.H., Rosebrough, N.J., Farr, A.L., Randall, R.J.: Protein measurement with the Folin phenol reagent. J. Biol. Chem., 1951; 193: 265-75.
11. Nelson, D.W., Sommers, L.E. Total carbon, organic carbon, and organic matter. In: Sparks, D.L. (Ed.), Methods of Soil Analysis. Part 3. Chemical Methods. SSSA Book Series no. 5. Soil Science Society of America \& American Society of Agronomy, Madison, WI, USA, 1996; 961-1010.

12. Hossain, S., Khalil, M.I., Alam, M.K., Khan, M.A., Alam, $N$. Upgrading of animal feed by solid state fermentation by Pleurotus sajor-caju. Eur. J. Appl. Sci., 2009; 1(4): 53-8.

13. Adamafio, N., Obodai, M., Brimpong, B. Solid state fermentation of maize (Zea mays) cob by Pleurotus ostreatus strain EM-1: Biopolymer profiles and cellulose degradability. Int. J. Biol. Chem. Sci., 2009; 3(6): 1459-66.

14. Issaka, J.H., Alemawor, F., Dzogbefia, V.P. Bioconversion Impact of Pleurotus ostreatus on the Value of Rice and Groundnut by-products as Feed Resources. Res. Biotechnol., 2013; 4(5):24-30.

15. Akinfemi, A., Adu, O.A., Doherty, F. Conversion of sorghum stover into animal feed with white-rot fungi: Pleurotus ostreatus and Pleurotus pulmonarius. Afr. J. Biotechnol.,2010; 9(11): 1706-12.

16. Bento, C.B.P., da Silva, J.S., Rodrigues, M.T., Kasuya, M.C.M., Mantovani, H.C. Influence of white-rot fungi on chemical composition and in vitro digestibility of lignocellulosic agro-industrial residues. Afr. J. Microbiol. Res., 2014; 8(28): 2724-32.

17. Shrivastava, B., Thakur, S., Khasa, Y.P., Gupte, A., Puniya, A.K., Kuhad, R.C. White-rot fungal conversion of wheat straw to energy rich cattle feed. Biodegradation.,2011; 22(4): 823-31. 